\title{
T-shaped Modified Delta Anastomosis as a Simple Intracorporeal Gastroduodenostomy
}

\author{
Chan Gyun Park, M.D., You Seong Yang, M.D., Jong Myeong Lee, M.D. \\ Department of Surgery, Presbyterian Medical Center, Jeonju, Korea
}

Purpose: A delta-shaped anastomosis (DA) is a widely accepted technique used for a totally laparoscopic distal gastrectomy (TLDG). Several studies have suggested various modifications to overcome the drawbacks of an original DA. We present our novel technique - a T-shaped modified delta anastomosis (TDA), and we report the early outcomes with its use in a case series.

Methods: We retrospectively reviewed the medical records of 40 patients who underwent a TLDG with TDA for early gastric cancer at $\mathrm{OOO}$ between February 2016 and May 2017. Perioperative outcomes, postoperative complications, and operating time were analyzed, and all data were expressed as means \pm standard deviation.

Results: We observed no major complications that required immediate postoperative intervention. Other minor and non-surgical complications were delayed gastric emptying $(n=1)$, pneumonia $(n=2)$, atelectasis $(n=3)$, dumping symptom $(n=1)$, and symptomatic bile reflux $(n=1)$. No wound infection was reported in any patient. The total operative time was $206.5 \pm 25.4 \mathrm{~min}$ and the estimated blood loss was $27.8 \pm 33.5 \mathrm{ml}$. The mean time required to perform the anastomosis was $20.9 \pm 6.7 \mathrm{~min}$, and the mean number of cartridges used during the operation was $4.78 \pm 0.66$.

Conclusion: We conclude that a TDA following a laparoscopic distal gastrectomy was successfully developed and showed acceptable clinical outcome.

Keywords: Laparoscopic surgery, Gastroduodenostomy, Intracorporeal anastomosis, Gastric cancer
Received September 2, 2017

Revised 1st October 16, 2017

2nd October 26, 2017

3rd November 6, 2017

Accepted November 7, 2017

Corresponding author

Jong Myeong Lee

Department of Surgery, Presbyterian Medical Center, 365 Seowon-ro, Wansan-gu, Jeonju 54987, Korea Tel: +82-63-230-1408

Fax: +82-63-230-1409

E-mail: gslee52kr@naver.com ORCID:

http://orcid.org/0000-0002-9985-7308

This is an Open Access article distributed under the terms of the Creative Commons Attribution Non-Commercial License (http:// creativecommons.org/licenses/by-nc/4.0/) which permits unrestricted non-commercial use, distribution, and reproduction in any medium, provided the original work is properly cited.

Copyright $\odot 2018$ The Journal of Minimally Invasive Surgery. All rights reserved.

\section{INTRODUCTION}

Gastroduodenostomy is the most commonly used anastomotic method following a distal gastrectomy, and its safety and feasibility are well known in Korea., ${ }^{1,2}$ Recently, the incidence of early gastric cancer has risen in Koreal and Japan, ${ }^{3}$ and the number of laparoscopic gastrectomies performed in Korea has been increasing. ${ }^{2}$ A laparoscopic gastrectomy is the treatment of choice for the management of early gastric cancer owing to several advantages over open surgery such as causing lesser postoperative pain, and better postoperative recovery, quality- of-life and cosmetic effects. ${ }^{4-7}$ Additionally, the feasibility of a laparoscopic gastrectomy for the treatment of even advanced gastric cancer has been reported in recent studies. ${ }^{89}$ Presently, a safe and feasible laparoscopic gastroduodenal anastomotic method needs to be established in this regard.

Laparoscopy-assisted distal gastrectomy (LADG) with $\mathrm{ex}^{-}$ tracorporeal gastroduodenostomy is the most commonly used technique in a laparoscopic distal gastrectomy because intracorporeal gastroduodenostomy is the most challenging step when performing a totally laparoscopic distal gastrectomy (TLDG). However, performing an intracorporeal anastomosis 
has become relatively easy since Kanaya et al. first introduced the intracorporeal delta-shaped anastomosis (DA) using only a linear stapler in 2002.10 Since then, TLDG with DA has been widely used, and its safety and feasibility have been proven. ${ }^{11-16}$

However, conventional delta anastomosis (cDA) is associated with certain drawbacks. A few authors have reported a risk of injury to the duodenal feeding vessels ${ }^{17}$ and an anastomotic leak because of the presence of 2 weak points where the staple lines are observed to cross. ${ }^{18}$ Furthermore, twisting the duodenum and the stomach during the cDA is technically difficult, for which modifications have been attempted. ${ }^{18-21}$ Noshiro et al. reported an anastomotic leak in 6 of 71 patients who underwent TLDG with DA, which was considered a technical drawback associated with a cDA. They developed a modification that involved performing an additional suture to prevent anastomotic leakage. ${ }^{19}$ Huang et al. dissected the duodenal cutting edge and performed an inverted T-shape modification whereby the intersection between staple lines was reduced to 1 , which was aimed at improving the safety of the anastomosis. ${ }^{18}$

At our hospital, we have attempted to perform a cDA; however, the risk of ischemia and the technical difficulty associated with the procedure necessitated the development of a $\mathrm{T}^{-}$ shaped modified delta anastomosis (TDA) to reduce the risk of anastomotic leakage and improve the repeatability of the procedure by simplifying the technique. A recent study reported by Jang et al. ${ }^{20}$ and Byun et al. ${ }^{21}$ is in agreement with ours in terms of minimizing the dissection of the posterior duodenal wall and simplifying the technique by performing the anastomosis between the anterosuperior wall of the duodenum and the greater curvature of the stomach without twisting. However, Jang et al. and Byun et al. anastomosed the duodenum after creating an orifice on the greater curvature of the stomach at a distance of proximal $6 \mathrm{~cm}$ from the stapling line of the remnant stomach, but we made it easier to approximate the stomach to the duodenum by creating the orifice over the posterior wall of the stomach at a distance of proximal $2 \mathrm{~cm}$, and we reduced the intersection between the staple lines to 1 to minimize the risk of anastomotic leakage.

We retrospectively investigated the short-term outcome of a TDA and assessed its acceptability. We introduce this method and report the results in this case series.

\section{MATERIALS AND METHODS}

\section{Patients}

We retrospectively reviewed the medical records of pa- $^{-}$ tients who underwent laparoscopic distal gastrectomy between February 2016 and May 2017 at Presbyterian Medical Center, Jeonju, Korea. During this period, 43 patients underwent the surgery. Patients who underwent a Billroth I anastomosis with TDA $(n=40)$ were included in this study, whereas 3 patients who underwent a Billroth II procedure with a Braun anastomosis were excluded. All surgical procedures were performed by a single surgeon (J.M.Lee). Patients underwent an endoscopic examination and computed tomography (CT) prior to surgery to determine their clinical stage and tumor location. In patients showing lesions located in the mid-third of the stomach, endoscopic clipping was performed in the proximal portion of the cancer prior to surgery, and radiographic imaging was performed to determine whether a Billroth I, Billroth II with Braun, or Roux-en-Y anastomosis should be performed. ${ }^{22}$ Patients in whom gallstones were detected on abdominal CT performed prior to surgery, or those demonstrating signs of chronic cholecystitis were informed that cholecystitis could worsen after gastrectomy; thus, a laparoscopic cholecystectomy was performed concomitantly with a gastrectomy in these patients after receiving their consent.

We retrospectively obtained information regarding: sex, age, body mass index (BMI), type of operation performed, the American Society of Anesthesiologists (ASA) score, intraoperative blood loss, patients who underwent a combined cholecystectomy, operative time, time required to perform the DA, the number of stapler cartridges used, the extent of lymph node (LN) dissection, the number of retrieved LNs, tumor size, tumor location, distal resection margin, proximal resection margin, pTNM stage, time until resumption of a soft diet, length of postoperative hospitalization, postoperative complications, mortality, and patients' symptoms. Clinical and pathological staging was performed based on the American Joint Committee on Cancer (AJCC) Staging Manual: 7th Edition. ${ }^{23}$

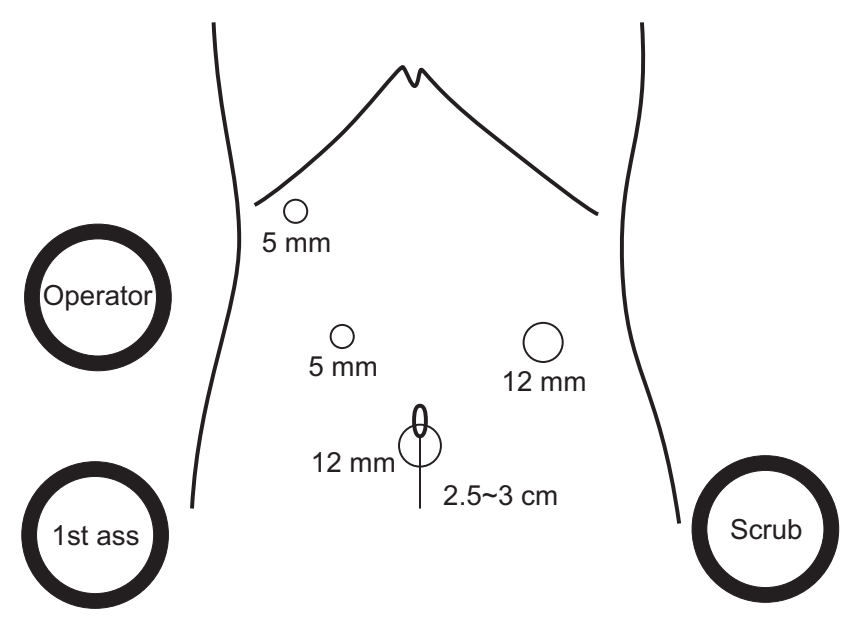

Fig. 1. Trocar placement for a totally laparoscopic distal gastrectomy (TLDG). 


\section{Surgical procedure}

The patient was placed in a reverse Trendelenburg position under general anesthesia, and 4 trocars were inserted (Fig. 1). The operator stood to the right side of the patient, a camera assistant stood to the right side of the operator, holding a camera in his left hand (a 30-degree camera was used), and assisting with his right hand. The role of the camera assistant was rather insignificant; he could provide assistance without having to be in the legs apart position as shown in the figure.

We began the procedure with adequate LN dissection. The extent of LN dissection was determined by the Japanese gastric cancer treatment guidelines 2010 (ver. 3). ${ }^{24}$ During dissection of the short gastric arteries, only 1 2 branches were dissected and the others were left intact.

During LN dissection, the liver was retracted without placement of an additional port. A monofilament nylon suture (Monosof ${ }^{\mathrm{TM}}$ ST 2 0) was fixed to the lesser omentum attached to the liver using a Hem-o-lok clip (Weck ${ }^{\circledR} \mathrm{Hem}^{-} \mathrm{O}^{-} \mathrm{lok}^{\circledR}$ ) to perform transabdominal traction.

\section{Intracorporeal gastroduodenostomy (T-shaped modified delta-shaped anastomosis procedure)}

A. Through a 12-mm port on the left lower side of the abdomen, we resected the duodenum leaving behind an adequate portion. The duodenum was divided in the dorsoventral direction using a $60 \mathrm{~mm}$ Endo-GIA stapler, similar to the procedure performed during a cDA. The duodenum was placed vertically, and a linear stapler was angulated to preserve a sufficient portion of the anterosuperior wall of the duodenal bulb (Fig. 2A).

B. We dissected adhesions around the duodenal stump, and additional dissection was performed on a portion of the retroperitoneal attachment to mobilize the duodenum. The stomach was gently pulled toward the duodenum to measure the distance. If the anticipated remnant stomach covered a small portion of the duodenum, it could be deduced that performing an anastomosis later would be easier.

C. A $60 \mathrm{~mm}$ Endo-GIA stapler was inserted to divide the stomach by firing this device twice (or thrice) from the side of the greater curvature toward the lesser curvature of the stomach. The initial firing of the stapler was perpendicular to the greater curvature (Fig. 2A).

D. After the stomach had been completely divided, an endopouch (LapBag U-Type) was inserted into the 12-mm port at the umbilicus to place the stomach and the resected LNs. The umbilical incision was extended vertically downward over a length of $2.5 \sim 3 \mathrm{~cm}$, and the specimen was removed. The stomach was opened and examined by visual analysis and

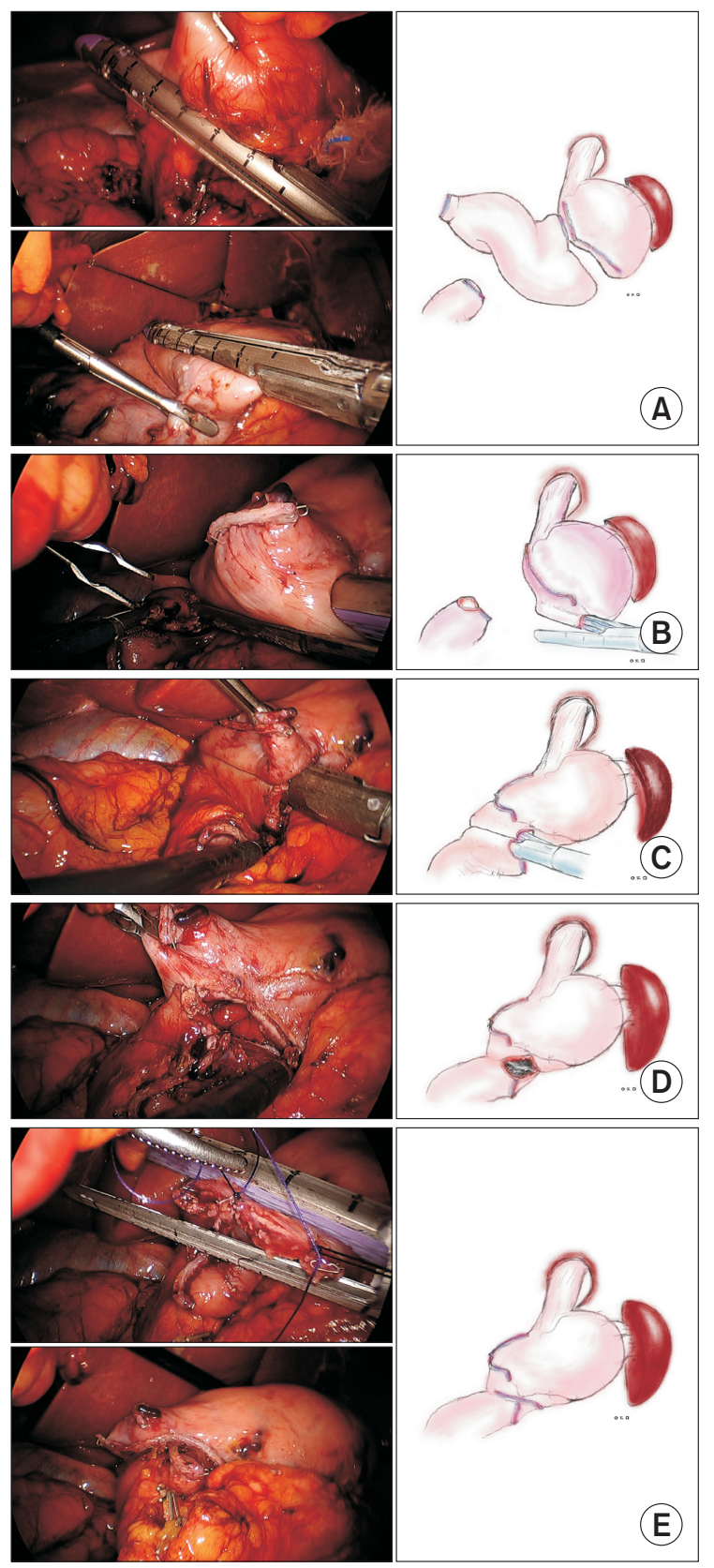

Fig. 2. (A) A totally laparoscopic distal gastrectomy (TLDG) with a Tshaped modified delta anastomosis (TDA) procedure. The duodenum is divided in the dorsoventral direction, as is performed for a cDA. The initial firing is observed to be perpendicular to the greater curvature of the stomach, and the stomach is completely divided. (B) A 1-cm orifice is created over the posterior wall of the greater curvature at a distance $2 \mathrm{~cm}$ from the staple line of the remnant stomach. The stapler jaw carrying the cartridge is inserted deep into the remnant stomach, and the posterior wall of the stomach is pulled toward the duodenum. (C) The cartridgefree jaw is inserted into the duodenal orifice. (D) Stapling is performed after accurately matching the edges of the 2 orifices. (E) A tagging suture is performed twice, and the completed anastomosis is observed to show a T-shaped appearance where the staple line intersects once. 
frozen section analysis to confirm that an adequate proximal resection margin had been obtained. In most cases it was easy to obtain a sufficient resection margin and perform an anastomosis during resection of early gastric cancer located in the distal half of the stomach.

E. After re-inflating the abdominal cavity, the remnant stomach and duodenum were pulled and crossed to check for possible excessive tension during the anastomosis. If the remnant stomach was determined to be smaller than expected and subsequent excessive tension was expected, a totally laparoscopic Billroth II procedure with Braun anastomosis was performed.

F. A 1-cm orifice was created by removing the staples on the anterior edge of the staple line on the duodenal stump. Another 1-cm orifice was created over the posterior wall of the greater curvature at a distance $2 \mathrm{~cm}$ from the staple line at the remnant stomach. This process is important to facilitate subsequent stapling. Next, a $45 \mathrm{~mm}$ Endo-GIA stapler was inserted into the $12-\mathrm{mm}$ port on the left lower side of the abdomen and inserted and advanced through the duodenal orifice until the thin jaw of the Endo-GIA without a cartridge reached the side of the duodenum. After removing the thin jaw from the duodenum, memorizing the angle of insertion, the thick jaw of the Endo-GIA with the cartridge was inserted deep into the remnant stomach, and the posterior wall of the remnant stomach was pulled toward the duodenum (Fig. 2B). The cartridge-free jaw was carefully inserted into the duodenal orifice, ensuring that the jaw carrying the cartridge was not lost in the remnant stomach (Fig. 2C). Complete insertion of staples was confirmed to prevent stenosis, and stapling was performed after accurately matching the edges of the two orifices (Fig. 2D).

G. The anastomotic site was carefully examined through a common stab incision to confirm that stapling had been appropriately and adequately performed and to check for bleeding. A tagging suture using Vicryl (VICRYL ${ }^{\mathrm{TM}}$ Plus 4-0) was performed twice to closely approximate the duodenal and stomach mucosa. Next, staple closure was performed using a $60 \mathrm{~mm}$ Endo-GIA stapler with minimal tissue dissection. This procedure eventually resulted in a T-shaped anastomosis with 1 intersecting staple line. (Fig. 2E) In a few patients, a VLoc (V-Loc ${ }^{\mathrm{TM}} 90$ 3-0) was used to laparoscopically perform a continuous running suture in double layers instead of staple closure.

H. After anastomosis was completed, the second portion of the duodenum was compressed with a device, and air was inflated into the stomach through a Levin tube to check if stomach distention could be well maintained and to rule out air leakage.

\section{Patient follow-up}

All patients underwent a postoperative follow-up examination at the outpatient clinic once a month over the first 3 months. Information was obtained from patients regarding any observed postoperative weight changes, the amount and frequency of food consumption, satiety or stomachache after meals, and heartburn and reflux symptoms. An upper gastrointestinal (UGI) series was performed 3 months postoperatively to assess food passage and to check for evidence of anas-

Table 1. Patient clinicopathological characteristics

\begin{tabular}{|c|c|}
\hline & $n=40$ \\
\hline Gender (male:female) & $25: 15(62.5 \%: 37.5 \%)$ \\
\hline Age (years) & $65.23 \pm 10.25$ \\
\hline $\mathrm{BM}^{*}\left(\mathrm{~kg} / \mathrm{m}^{2}\right)$ & $24.98 \pm 3.55$ \\
\hline Tumor size (cm) & $2.25 \pm 1.07$ \\
\hline \multicolumn{2}{|l|}{ Tumor location } \\
\hline Lower third & $28(70 \%)$ \\
\hline Middle third & $12(30 \%)$ \\
\hline \multicolumn{2}{|l|}{ pT stage } \\
\hline T1a & $21(52.5 \%)$ \\
\hline $\mathrm{T} 1 \mathrm{~b}$ & 17 (42.5\%) \\
\hline $\mathrm{T} 2$ & $2(5.0 \%)$ \\
\hline T3 & $0(0.0 \%)$ \\
\hline \multicolumn{2}{|l|}{ pN stage } \\
\hline NO & $35(87.5 \%)$ \\
\hline N1 & $5(12.5 \%)$ \\
\hline N2 & $0(0.0 \%)$ \\
\hline N3 & $0(0.0 \%)$ \\
\hline \multicolumn{2}{|l|}{ pTNM stage } \\
\hline la & $35(87.5 \%)$ \\
\hline $\mathrm{lb}$ & $3(7.5 \%)$ \\
\hline Ila & $2(5.0 \%)$ \\
\hline Ilb & $0(0.0 \%)$ \\
\hline \multicolumn{2}{|l|}{$\mathrm{ASA}^{\dagger}$ classification } \\
\hline 1 & $5(12.5 \%)$ \\
\hline 2 & $19(47.5 \%)$ \\
\hline 3 & $16(40.0 \%)$ \\
\hline
\end{tabular}

Data are means \pm standard deviation or numbers (percentages) unless otherwise indicated. ${ }^{*} \mathrm{BMI}=$ body mass index ${ }^{\dagger} \mathrm{ASA}=$ American Society of Anesthesiologists. 
tomotic stenosis. An esophagogastroduodenoscopy (EGD) was performed 1 year postoperatively to evaluate for recurrence, as well as the status of the anastomotic site and the presence of gastroesophageal reflux disease (GERD).

\section{RESULTS}

Between February 2016 and May 2017, 40 patients underwent TLDG with TDA, and all patients underwent R0 resection. No patient required conversion from laparoscopic to open distal gastrectomy. Patients who underwent a Billroth II procedure with Braun anastomosis because they were suspected to demonstrate excessive tension at the anastomotic site during surgery excluded from this study.

Of the 40 patients studied, $25(62.5 \%)$ were men and 15 (37.5\%) were women. The mean age was $65.23 \pm 10.25$ years, and the mean BMI was $24.98 \pm 3.55 \mathrm{~kg} / \mathrm{m}^{2}$. The mean tumor size was $2.25 \pm 1.07 \mathrm{~cm}$. We observed that the ASA score was 1 in 5 (12.5\%), 2 in 19 (47.5\%), and 3 in 16 (40\%) patients, respectively. Tumor location, $\mathrm{pT}$ stage, $\mathrm{pN}$ stage and pTNM stage are shown in Table 1.

The intraoperative blood loss was $27.8 \pm 33.5 \mathrm{ml}$. A concomitant cholecystectomy was performed in 9 patients (22.5\%), a D1+ dissection in 18 patients (45.0\%), and a D2 dissection in 22 patients (55.0\%). The number of retrieved LNs was $37.9 \pm$ 12.0. The proximal resection margin measured $4.69 \pm 2.44 \mathrm{~cm}$, and the distal resection margin measured $5.33 \pm 2.25 \mathrm{~cm}$. Postoperatively, time until resumption of a soft bland diet was 5.18 \pm 0.45 days, and length of hospitalization was $10.60 \pm 5.11$ days (Table 2).

No postoperative mortality was observed in any patient

Table 2. Perioperative outcomes

\begin{tabular}{lc}
\hline & $\mathrm{n}=40$ \\
\hline Blood loss (ml) & $27.8 \pm 33.5$ \\
\hline Combined cholecystectomy (no. of patients, \%) & $9(22.5 \%)$ \\
$\begin{array}{l}\text { Lymph node dissection } \\
\text { D1 }+\alpha / \beta\end{array}$ & $18(45.0 \%)$ \\
D2 & $22(55.0 \%)$ \\
Retrieved lymph node & $37.9 \pm 12.0$ \\
\hline Proximal resection margin (cm) & $4.69 \pm 2.44$ \\
\hline Distal resection margin (cm) & $5.33 \pm 2.25$ \\
\hline SBD* start day & $5.18 \pm 0.45$ \\
\hline Discharge day & $10.60 \pm 5.11$ \\
\hline
\end{tabular}

Data are means \pm standard deviation or numbers (percentages) unless otherwise indicated. ${ }^{*}$ SBD $=$ soft bland diet. who underwent a laparoscopic distal gastrectomy during the study period, nor was postoperative anastomotic leakage reported. We observed that an anastomotic stricture developed in 1 patient (2.5\%), postoperative bleeding was not reported in any patient, and an intra-abdominal abscess was reported in 1 patient $(2.5 \%)$. Other postoperative complications were: $1 \mathrm{pa}^{-}$ tient showed delayed gastric emptying (2.5\%), 2 showed pneumonia (5.0\%), 3 showed atelectasis $(7.5 \%), 0$ showed ileus, 1 showed dumping symptom (2.5\%), 1 showed symptomatic bile reflux $(2.5 \%)$ and 0 patients showed wound infection. (Table 3) Delayed gastric emptying was defined as the development of signs of gastrostasis detected on abdominal radiography during the process of diet build-up, necessitating fasting due to discomfort after eating. Symptomatic GERD was defined as the development of heartburn, and signs of bile reflux observed on a UGI series or EGD.

The total operative time for a TLDG with TDA was 206.5

Table 3. Postoperative complications

\begin{tabular}{|ll|}
\hline & $\mathrm{n}=40$ \\
\hline Motality (no. of patients, \%) & 0 \\
\hline Anastomotic leakage & 0 \\
\hline Anastomotic stricture & $1(2.5 \%)$ \\
\hline Post operative bleeding & 0 \\
\hline Intra-abdominal abscess & $1(2.5 \%)$ \\
\hline Delayed gastric emptying & $1(2.5 \%)$ \\
\hline Pneumonia & $2(5.0 \%)$ \\
\hline Atelectasis & $3(7.5 \%)$ \\
\hline Ileus & 0 \\
\hline Dumping symptom & $1(2.5 \%)$ \\
\hline Symptomatic reflux & $1(2.5 \%)$ \\
\hline Wound infection & $0(0.0 \%)$ \\
\hline
\end{tabular}

Data are numbers (percentages) unless otherwise indicated.

Table 4. Operative data

\begin{tabular}{|c|c|}
\hline & $\mathrm{n}=40$ \\
\hline Operative time $(\mathrm{min})$ & $206.5 \pm 25.4$ \\
\hline Anastomosis time $(\mathrm{min})$ & $20.9 \pm 6.7$ \\
Suture & $28.1 \pm 6.7$ \\
Stapling & $17.9 \pm 3.9$ \\
\hline Used cartridges number & $4.78 \pm 0.66$ \\
\hline
\end{tabular}

Data are means \pm standard deviation. 
$\pm 25.4 \mathrm{~min}$, and the mean time required for the creation of a TDA was $20.9 \pm 6.7 \mathrm{~min}$. The mean time required for creation of the anastomosis in those who underwent suturing was 28.1 $\pm 6.7 \mathrm{~min}$, and in those who underwent stapling it was $17.9 \pm 3.9$ min (Table 4). The mean number of cartridges used during the TDA was 4.78 \pm 0.66 .

A UGI series performed 3 months postoperatively showed a wide anastomosis, which enabled smooth passage of food (Fig. 3A). Anastomotic sites appeared circular on an EGD examination (Fig. 3B).

\section{DISCUSSION}

Lately, a DA is a widely accepted procedure for a TLDG..$^{11-14}$ Recent studies have shown that TLDG with DA offers several advantages over the LADG procedure. Kim et al. have reported that because the stomach is not pulled through a minilaparotomy during a DA, injuries that can occur secondary to excessive tension during LADG can be avoided, and good visualization of the operative field can be obtained, making DA a favorable option for patients with obesity. Additionally, bleeding can be controlled more effectively than during a circular stapler anastomosis in LADG. ${ }^{25}$ Song et al. have reported that TLDG is a less invasive procedure than LADG and that it results in faster bowel recovery. ${ }^{12} \mathrm{~A}$ recent meta-analysis that described TLDG and LADG has reported that TLDG with DA is safer and more feasible than LADG, and that it offers the advantage of significantly reduced blood loss, earlier resumption of oral intake, and a shorter postoperative hospitalization period. $^{13}$

However, cDA is associated with a few drawbacks. Kitakami et al. have reported that duodenal ischemia can occur during the preparation process, which involves dissection of the tissues surrounding the posterior wall of the duodenum prior to stapling. Furthermore, the $45 \mathrm{~mm}$ stapling process can induce ischemia as it involves dissection of the feeding vessels supplying the duodenum. ${ }^{17}$ Huang et al. have reported that because of an anastomotic intersection where 2 staple lines intersect, it could compromise the blood supply to these sites leading to anastomotic site complications. ${ }^{18}$ Furthermore, Park et al. have reported that surgical costs increase due to the use of a larger number of stapler cartridges. ${ }^{26}$

In order to overcome these limitations, we developed the TDA procedure and assessed the incidence of anastomosisrelated complications such as leakage, stenosis, and bleeding associated with this technique. We observed no postoperative bleeding or leakage in any patient, and anastomotic site stenosis occurred in only 1 patient who underwent TLDG with TDA. In this patient, although stapling was performed, the 45 $\mathrm{mm}$ staplers had not been completely inserted due to excessive tension between the stomach and duodenum. This patient was transferred to another hospital while he received conservative treatment and was considered a candidate for balloon dilatation. The key take-away from this case was that complete insertion of the $45 \mathrm{~mm}$ stapler is extremely important to prevent TDA stenosis. Moreover, it is important to ensure that the mucosal edges are well-approximated during common stab incision closure to achieve minimal tissue dissection.

No major complications requiring immediate postoperative intervention were reported, and the frequency of minor complications such as wound infection $(\mathrm{n}=0)$, delayed gastric emptying $(n=1)$, pneumonia $(n=2)$, and atelectasis $(n=3)$ was acceptable. The incidence of dumping symptom $(n=1)$ and symptomatic bile reflux $(n=1)$, which were evaluated during follow-up period, was also acceptable.

We performed a double layer running suture using a V-Loc if during common stab incision closure we detected an inadequate approximation between the stomach and duodenal mucosa. We usually performed the TDA with a running suture in the early period after the introduction of the TDA. However,
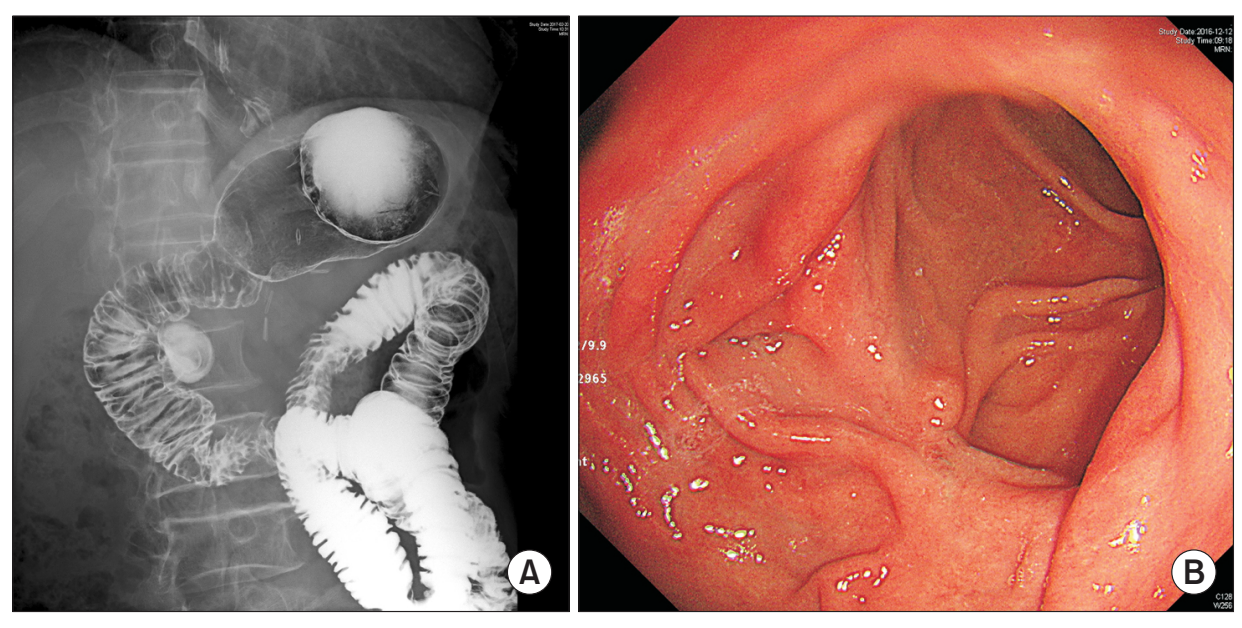

Fig. 3. (A) Images of an upper gastrointestinal (UGI) series performed 3 months postoperatively showing a wide anastomosis. (B) Images showing that the anastomotic sites appear circular on esophagogastroduodenoscopic (EGD) examination. 
as surgeons gained more experience, closure could be performed using a single $60 \mathrm{~mm}$ linear stapler in the later period. TDA was performed more rapidly when a stapler was used instead of a suture (17.9 \pm 3.9 min vs. $28.1 \pm 6.7$ min, respectively). The mean time required for TDA was $20.9 \pm 6.7 \mathrm{~min}$, and this procedural time is expected to reduce as frequency of the TDA with stapling increases in the future.

We used a total of 5 cartridges during TLDG with TDA. Because no twisting was observed during the anastomosis operation, the common stab incision did not get twisted, and staplers were inserted into a small incision $(1 \mathrm{~cm})$, and closure of the small common stab incision could be achieved using a single $60 \mathrm{~mm}$ cartridge. Several studies in which cDA was performed have reported the use of more than 6 cartridges per operation on average. ${ }^{25,27}$ Our method involves the use of fewer stapler cartridges, the cost for the operation could be reduced.

Limitations of our study: 1) Ours was a retrospective study, and we did not compare our results with those of a cDA operation. 2) We could not evaluate the rates of delayed complications such as bile reflux and gastritis because of the short follow-up period. 3) We investigated the incidence of complications based on a subjective assessment of patient-reported outcomes (dumping symptom or GERD). Thus, we could not objectively assess the incidence of complications.

We developed the TDA procedure to overcome the drawbacks associated with cDA. Our technique creates an anastomosis between the anterior duodenal wall and the posterior wall of the stomach without twisting of organs, which therefore reduces the risk of anastomotic leakage and simplifies the procedure. This retrospective study involving patients who underwent TLDG with TDA at our hospital showed that the short-term results including the occurrence of postoperative complications were acceptable. However, further studies and experience are warranted to evaluate the long-term results.

\section{REFERENCES}

1) Information Committee of Korean Gastric Cancer Association. Korean Gastric Cancer Association Nationwide Survey on Gastric Cancer in 2014. J Gastric Cancer 2016;16:131-140.

2) Jeong O, Park YK. Clinicopathological features and surgical treatment of gastric cancer in South Korea: the results of 2009 nationwide survey on surgically treated gastric cancer patients. J Gastric Cancer 2011;11:69-77.

3) Kitano S, Shiraishi N, Uyama I, Sugihara K, Tanigawa N. A multicenter study on oncologic outcome of laparoscopic gastrectomy for early cancer in Japan. Ann Surg 2007;245:68-72.

4) Adachi $Y$, Shiraishi N, Shiromizu A, Bandoh T, Aramaki M, Kitano S. Laparoscopy-assisted Billroth I gastrectomy compared with conventional open gastrectomy. Arch Surg 2000;135:806-810.
5) Kim YW, Baik YH, Yun YH, et al. Improved quality of life outcomes after laparoscopy-assisted distal gastrectomy for early gastric cancer: results of a prospective randomized clinical trial. Ann Surg 2008;248:721-727.

6) Kitano S, Shiraishi N, Kakisako K, Yasuda K, Inomata M, Adachi Y. Laparoscopy-assisted Billroth-I gastrectomy (LADG) for cancer: our 10 years' experience. Surg Laparosc Endosc Percutan Tech 2002;12:204-207.

7) Lee JH, Han HS, Lee JH. A prospective randomized study comparing open vs laparoscopy-assisted distal gastrectomy in early gastric cancer: early results. Surg Endosc 2005;19:168-173.

8) Park EM, Kim HO, Son BH, Shin JH, Lee SR. Safety of Laparoscopy Assisted Gastrectomy for Gastric Cancer, Including Advanced Cancers. J Minim Invasive Surg 2015;18:79-85.

9) An H, Cho H, Kim HJ, et al. Feasibility of Laparoscopic D2 Lymph Node Dissection in Gastric Cancer. J Minim Invasive Surg 2012;15:121-125.

10) Kanaya S, Gomi T, Momoi H, et al. Delta-shaped anastomosis in totally laparoscopic Billroth I gastrectomy: new technique of intraabdominal gastroduodenostomy. J Am Coll Surg 2002;195:284287.

11) Ikeda O, Sakaguchi Y, Aoki Y, et al. Advantages of totally laparoscopic distal gastrectomy over laparoscopically assisted distal gastrectomy for gastric cancer. Surg Endosc 2009;23:2374-2379.

12) Song KY, Park CH, Kang HC, et al. Is totally laparoscopic gastrectomy less invasive than laparoscopy-assisted gastrectomy?: prospective, multicenter study. J Gastrointest Surg 2008;12:10151021.

13) Hu GY, Tao F, Ji KW, Wang W. Comparison of Delta-Shape Anastomosis and Extracorporeal Billroth I Anastomosis after Laparoscopic Distal Gastrectomy for Gastric Cancer: A Systematic Review with Meta-Analysis of Short-Term Outcomes. PLoS One 2016;11:e0162720.

14) Lee HH, Song KY, Lee JS, Park SM, Kim JJ. Delta-shaped anastomosis, a good substitute for conventional Billroth I technique with comparable long-term functional outcome in totally laparoscopic distal gastrectomy. Surg Endosc 2015;29:2545-2552.

15) Kim DG, Choi YY, An JY, et al. Comparing the short-term outcomes of totally intracorporeal gastroduodenostomy with extracorporeal gastroduodenostomy after laparoscopic distal gastrectomy for gastric cancer: a single surgeon's experience and a rapid systematic review with meta-analysis. Surg Endosc 2013;27:31533161.

16) Wang SY, Hong J, Hao HK. A comparative study of delta-shaped and conventional Billroth I anastomosis after laparoscopic distal gastrectomy for gastric cancer. Surg Endosc 2017;31:3191-3202.

17) Kitagami $H$, Morimoto $M$, Nozawa $M$, et al. Evaluation of the delta-shaped anastomosis in laparoscopic distal gastrectomy: midterm results of a comparison with Roux-en-Y anastomosis. Surg Endosc 2014;28:2137-2144. 
18) Huang CM, Lin M, Lin JX, et al. Comparision of modified and conventional delta-shaped gastroduodenostomy in totally laparoscopic surgery. World J Gastroenterol 2014;20:10478-10485.

19) Noshiro H, Iwasaki H, Miyasaka $Y$, et al. An additional suture secures against pitfalls in delta-shaped gastroduodenostomy after laparoscopic distal gastrectomy. Gastric Cancer 2011;14:385-389.

20) Jang CE, Lee SI. Modified intracorporeal gastroduodenostomy in totally laparoscopic distal gastrectomy for gastric cancer: early experience. Ann Surg Treat Res 2015;89:306-312.

21) Byun C, Cui LH, Son SY, Hur H, Cho YK, Han SU. Linearshaped gastroduodenostomy (LSGD): safe and feasible technique of intracorporeal Billroth I anastomosis. Surg Endosc 2016;30:4505-4514.

22) Choi EH, Lee JM. Evaluation of Different Methods of Gastroenterostomy after Distal Gastrectomy for Gastric Carcinoma. J Korean Gastric Cancer Assoc 2009;9:215-222.

23) Edge SB, American Joint Committee on Cancer. AJCC cancer staging manual. 7th ed. New York: Springer; 2010.

24) Japanese Gastric Cancer Association. Japanese gastric cancer treatment guidelines 2010 (ver. 3). Gastric Cancer 2011;14:113123.

25) Kim JJ, Song KY, Chin HM, et al. Totally laparoscopic gastrectomy with various types of intracorporeal anastomosis using laparoscopic linear staplers: preliminary experience. Surg Endosc 2008;22:436-442.

26) Park SK, Lee HH, Kim JJ, Park SM. Delta-Shaped Gastroduodenostomy after Totally Laparoscopic Distal Gastrectomy: A Comparison Analysis between Early and Late Experience. J Korean Surg Soc 2010;79:110-115.

27) Lee HW, Kim HI, An JY, et al. Intracorporeal Anastomosis Using Linear Stapler in Laparoscopic Distal Gastrectomy: Comparison between Gastroduodenostomy and Gastrojejunostomy. J Gastric Cancer 2011;11:212-218. 